\title{
Natural Products that Target the NLRP3 Inflammasome to Treat Fibrosis
}

OPEN ACCESS

Edited by:

Annalisa Bruno,

University of Studies G. d'Annunzio

Chieti and Pescara, Italy

Reviewed by:

Louise Bennett,

Monash University, Australia

Ulf Simonsen,

Aarhus University, Denmark

Takanori Komada,

University of Calgary, Canada

*Correspondence:

Yimou Wu

yimouwu@sina.com

Specialty section:

This article was submitted to Inflammation Pharmacology,

a section of the journal

Frontiers in Pharmacology

Received: 04 August 2020 Accepted: 10 November 2020

Published: 17 December 2020

Citation:

Ding N, Wei B, FuX, Wang C and Wu Y (2020) Natural Products that Target the NLRP3 Inflammasome to

Treat Fibrosis.

Front. Pharmacol. 11:591393.

doi: 10.3389/fphar.2020.591393

\author{
Nan Ding ${ }^{1}$, Bo Wei ${ }^{2}$, Xiaohui $F u^{1}$, Chuan Wang ${ }^{1}$ and Yimou $W^{1 *}$ \\ ${ }^{1}$ Institute of Pathogenic Biology, Hengyang Medical College, Hunan Provincial Key Laboratory for Special Pathogens Prevention \\ and Control, Hunan Province Cooperative Innovation Center for Molecular Target New Drug Study, University of South China, \\ Hengyang, China, ${ }^{2}$ Research lab of Translational Medicine, Hengyang Medical College, University of South China, Hengyang, \\ China
}

Fibrosis is a common pathway followed by different organs after injury, and it can lead to parenchymal scarring, cellular dysfunction, and even organ failure. The NLRP3 inflammasome is a multiprotein complex composed of the sensor molecule NLRP3, the adaptor apoptosis-associated speck-like protein containing a CARD (ASC), and the effector protease caspase-1. Overactivation of the NLRP3 inflammasome triggers the abundant secretion of IL-1 $\beta$ and IL-18, induces pyroptosis, and promotes the release of a swathe of proinflammatory proteins, all of which contribute to fibrogenic processes in multiple organs. In recent years, screening bioactive natural compounds for NLRP3 inhibitors to alleviate fibrosis has gained broad interest from the scientific community because of the associated cost-effectiveness and easy access. In this review, we systematically and comprehensively summarize the natural products, including terpenoids, phenols, and alkaloids, among others, and the plant-derived crude extracts, that have been reported to ameliorate fibrosis via inhibiting NLRP3 inflammasome activation and highlight the underlying mechanisms. Among all the compounds, diterpenoids is the most promising candidates for inhibiting NLRP3 inflammasome activation and improving fibrosis, as they possess combined inhibitory effect on NLRP3 inflammasome assembly and NF-кB signaling pathway. All the information may aid in the development of therapeutic strategies for the treatment of fibrotic diseases.

Keywords: herbs, phytochemicals, fibrosis, NLRP3 inflammasome, terpenoids, phenols

\section{INTRODUCTION}

Fibrosis refers to the excessive accumulation of extracellular matrix (ECM) due to persistent or severe tissue injury, aging, or genetic factors that can lead to permanent scarring, organ dysfunction, and even death (Noronha et al., 2002; Wynn and Ramalingam, 2012). Many conditions, including liver, kidney, heart, and lung disorders, chronic autoimmune diseases, and tumors, would ultimately suffer from the pathological manifestation of fibrosis without reasonable intervention.

Abnormal activation of the NLRP3 inflammasome is an important contributor to inflammatory disease and subsequent fibrosis. Numerous reviews have systematically clarified the pivotal role played by the NLRP3 inflammasome in fibrogenic disorders, including those of the liver, kidney, heart, and lung (Pelegrin et al., 2017; Kim et al., 2019; Pinar et al., 2020), which suggests that targeting the NLRP3 inflammasome has potential as a therapeutic strategy to treat fibrosis. 
The synthetic compound MCC950 (also known as CRID3 and CP 456,773) is the most studied NLRP3 inhibitor, which specifically suppresses NLRP3 inflammasome assembly to block all NLRP3 activation pathways. However, although MCC950 displays significant efficacy in various NLRP3dependent murine disease models, the phase-II clinic trials for rheumatoid arthritis are unsuccessful owning to elevated serum liver enzyme levels in the clinic (Mangan et al., 2018). Therefore, screening NLRP3 inflammasome inhibitors with low toxicity from natural compounds has become an effective means of identifying drugs to treat inflammation and fibrosis. In this review, we aim to summarize the natural products, including terpenoids, phenols, and alkaloids, among others, that target the NLRP3 inflammasome to ameliorate fibrosis both in vivo and in vitro. This information may aid in the development of novel strategies for the discovery of antifibrotic drugs.

\section{THE PROCESS OF FIBROSIS}

The occurrence of fibrosis can be divided into four major phases (Rockey et al., 2015). The first is response initiation, during which primary injury occurs and proinflammatory factors are released by injured epithelial and/or endothelial cells. This is followed by the activation of effector cells, during which chemokines and growth factors, such as matrix metalloproteinases (MMPs), interleukin (IL)-13, and transforming growth factor beta 1 (TGF- $\beta 1$ ), are secreted. The next phase comprises the elaboration of the ECM, during which myofibroblasts, the key effector cells, proliferate and synthesize abundant ECM under pathological conditions, eventually leading to excessive accumulation of collagen, which represents the fourth phase of fibrosis (Strutz, 1995; Noronha et al., 2002; Rockey et al., 2015). In summary, the essence of fibrosis is the activation of quiescent fibroblasts and the induction of ECM production and accumulation.

ECM is made of various proteins, proteoglycans, and glycosaminoglycans, including the main components collagen and fibronectin. The deposition of ECM depends on the balance between ECM synthesis and degradation. MMPs, the main enzymes responsible for ECM degradation, are able to cleave ECM components with wide substrate specificities. The activity of MMPs is relatively low in normal conditions but improved during repairing or remodeling processes and in diseased or inflamed tissue. The tissue inhibitor of the metalloproteinases (TIMP) family are the main components that reversibly inhibit the activity of MMPs, thus avoiding excessive and deleterious tissue degradation (Bonnans et al., 2014).

\section{THE NLRP3 INFLAMMASOME AND FIBROSIS}

Acute and chronic inflammation are the main fibrosis-triggering factors. Resident epithelial or endothelial cells are often injured under inflammatory conditions, leading to enhanced release of mediators of inflammation, such as cytokines and chemokines.
These factors induce the recruitment of a variety of inflammation-related cells, such as macrophages, lymphocytes, eosinophils, and basophils, that activate fibroblasts and myofibroblasts, thereby leading to fibrosis (Rockey et al., 2015).

The NLRP3 inflammasome is the most widely studied of the inflammasomes. It is composed of the sensor molecule NLRP3, the adaptor ASC (apoptosis-associated speck-like protein containing a CARD), and the effector protease caspase-1 (procaspase-1). The NLRP3 inflammasome can recognize pathogen-associated molecular patterns (PAMPs) and dangerassociated molecular patterns (DAMPs), which triggers the cleavage of dormant procaspase- 1 to generate active caspase-1, further converting the cytokine precursors pro-IL- $1 \beta$ and pro-IL18 into mature and biologically active IL- $1 \beta$ and IL-18, respectively (Schroder and Tschopp, 2010). In addition, the caspase-1 also can cleave the gasdermin D (GSDMD), a complex composed of an amino-terminal cell death domain $\left(G_{S D M D}{ }^{\text {Nterm }}\right)$, a central short linker region, and a carboxyterminal autoinhibition domain, to release the GSDMD ${ }^{\mathrm{Nterm}}$, which combines with phosphatidylserine and phosphatidylinositol phosphates in the cell membrane inner leaflet, further forming a $10-14 \mathrm{~nm}$ pore containing 16 symmetrical protomers, thus leading to the death of cells and excessive release of inflammatory mediators, including IL- $1 \beta$ and IL-18 (Swanson et al., 2019).

The expression of NLRP3 and associated proteins is markedly enhanced in the organs of fibrosis patients (Wree et al., 2014; Ke et al., 2018). Persistent activation of the NLRP3 inflammasome leads to the abundant secretion of IL- $1 \beta$ and IL-18 and initiation of pyroptosis, all of which are major contributors to the formation of fibrotic lesions (Pinar et al., 2020). IL-1 $\beta$ is a potential mediator of the crosstalk between inflammation and fibrosis and can generate a positive feedback cycle to dominate the secretory cytokine profile in cystic fibrosis (Otto, 2018; Chen et al., 2019). Additionally, the deletion or inhibition of IL-18 can alleviate cardiac or renal fibrosis in corresponding mouse models (Liang et al., 2018; Xiao et al., 2018). Several mechanisms underly how IL-1 $\beta$, IL-18, and pyroptosis enhance inflammation and fibrosis after inflammasome activation. First, secreted IL- $1 \beta$ and IL-1 8 bind to the IL- 1 receptor, type I (IL-1RI) and IL-18R, which further activates the nuclear factor kappa B $(\mathrm{NF}-\kappa \mathrm{B})$ signaling pathway to induce the expression of multiple inflammation-related proteins. Second, following the release of proteins such as caspase-1 and nuclear protein high-mobility group box 1 (HMGB1) into the extracellular space, the immune system will detect the presence of these types of proteins and activate inflammatory responses. Moreover, the occurrence of pyroptosis promotes the release of various protein complexes, especially those comprising the NLRP3 inflammasome. On the one hand, extracellularly localized inflammasomes can activate caspase-1 owing to the presence of high concentrations of procaspase- 1 in the extracellular milieu, thereby inducing a series of inflammatory responses. On the other hand, macrophages can internalize extracellular inflammasomes, thus further promoting caspase-1 activation (Baroja-Mazo et al., 2014; Pelegrin et al., 2017). The enhanced secretion of IL-1 $\beta$ is able to downregulate MMPs, upregulate TIMPs and promote the 


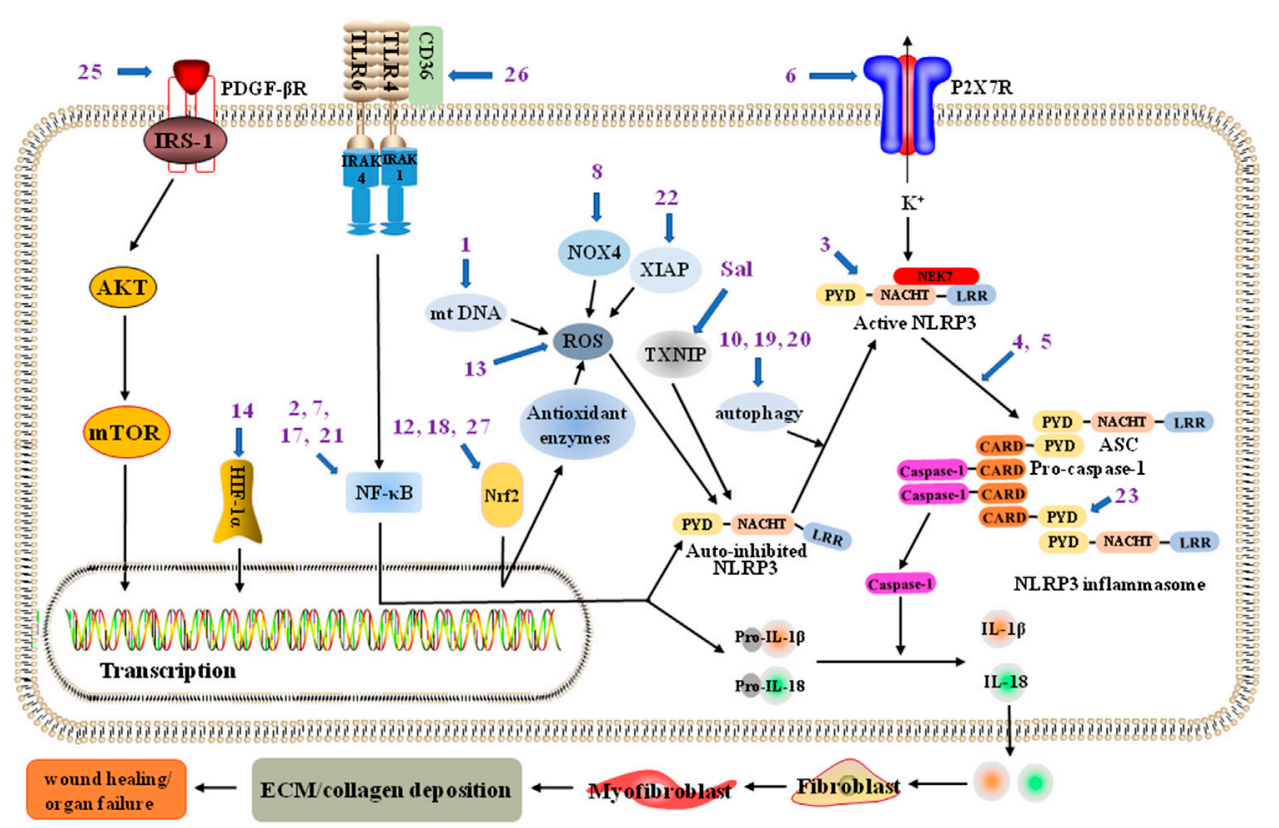

FIGURE 1 | The NLRP3 inflammasome pathway associated with potential blockade sites of various pharmacological inhibitors to treat fibrosis. NLRP3 (NOD-, LRR- and pyrin domain-containing 3) inflammasome in liver fibsosis could be inhibited by NLRP3 inflammasome assembly inhibitors andrographolide (4) and oridonin (3, targeting the NACHT domain in NLRP3), mitochondrial DNA (mt DNA) de novo synthesis inhibitor sweroside (1), P2X purinoceptor 7 (P2X7) inhibitor 25-OCH ${ }_{3}-\mathrm{PPD}(\mathbf{6})$, Nrf2 pathway promotor quercetin (12) and pelargonidin (18), and platelet-derived growth factor- $\beta$ receptor (PDGF- $\beta$ R) inhibitor tetramethylpyrazine (25); NLRP3 inflammasome in renal fibsosis could be inhibited by nuclear factor (NF)-KB pathway inhibitors artemisinin (2), compound K (7), and icariin (17), Nrf2 pathway promotor astaxanthin (27), nicotinamide adenine dinucleotide phosphate oxidase 4 (NOX4) inhibitor ginsenoside Rg1 (8), ROS production inhibitor dihydroquercetin (13), autophagy activator pterostilbene (20), and X-linked inhibitor of apoptosis protein (XIAP) suppressant (22); NLRP3 inflammasome in cardiac fibsosis could be inhibited by NLRP3 inflammasome assembly inhibitor triptolide (5), CD36 inhibitor cinnamaldehyde (26), and TXNIP suppressant of crude extract salvianolates (Sal); NLRP3 inflammasome in pulmonary fibsosis could be inhibited by NLRP3 inflammasome assembly inhibitor lycorine (23, targeting the PYD of ASC), autophagy activator resveratrol (19), and NF-kB pathway inhibitor polydatin (21); NLRP3 inflammasome in synovial tissue fibsosis could be inhibited by hypoxia-inducible factors (HIF)-1a pathway inhibitor casticin (14); NLRP3 inflammasome in pancreatic fibrosis might be inhibited by autophagy activator saikosaponin A (10).

production of collagen I (Pinar et al., 2020). IL-18 also has been shown to possess an 8 -fold enhancement in interstitial collagen content, leading to increased ECM accumulation and fibrogenesis (O'|'Brien et al., 2014). Additionally, the augmented inflammasome-independent NLRP3, IL-1 $\beta$ and IL-18 all can activate the mitogen-activated protein kinase (MAPK) signaling pathway to upregulate the expression of TGF- $\beta 1$ and induce fibroblast differentiation and collagen production (Artlett, 2012; Wang et al., 2013).

\section{NLRP3 INFLAMMASOME ACTIVATION}

NLRP3 inflammasome activation consists of two steps: priming and activation. In the resting state, the amount of NLRP3 present in the cell is insufficient to allow inflammasome activation. In the priming step, the NF- $\mathrm{B}$ pathway is activated by pattern recognition receptors (PRRs) such as Toll-like receptors (TLRs), nucleotide-binding oligomerization domain-containing protein 2 (NOD2), and the granulocyte-macrophage colony stimulating factor (GM-CSF) receptor; this leads to the upregulation of the transcription and expression of NLRP3 and other key proinflammatory components, including pro-IL$1 \beta$ and pro-IL-18 (Shao et al., 2019). The expressed NLRP3 undergoes autoinhibition due to phosphorylation, dephosphorylation, ubiquitination, or alkylation modifications, which work together to regulate NLRP3 degradation and selfassociation. NLRP3 is subsequently activated by processes such as $\mathrm{K}^{+}$efflux, $\mathrm{Ca}^{2+}$ flux, $\mathrm{Cl}^{-}$efflux, lysosomal disruption, and mitochondrial dysfunction (ROS production), which triggers the assembly of the NLRP3, ASC, and procaspase-1 proteins into the NLRP3 inflammasome. Mature caspase-1 is then released, resulting in further rapid processing of pro-IL- $1 \beta$ and pro-IL-18 into biologically active IL- $1 \beta$ and IL-18, respectively (Swanson et al., 2019) (Figure 1). Additionally, the NLRP3 inflammasome can also be activated via a noncanonical signaling pathway in which caspase-11cleave the GSDMD and induce pyroptosis, thus promoting $\mathrm{K}^{+}$efflux and the release of IL$1 \beta$ (Kayagaki et al., 2015; Wang et al., 2020a).

\section{PHYTOCHEMICALS THAT SUPPRESS THE NLRP3 INFLAMMASOME AND INHIBIT FIBROSIS}

Screening for NLRP3 inhibitors from natural products to treat fibrosis has gained broad interest from the scientific community. Numerous studies have reported that natural products, including 
<smiles>C=CC1[C-](OC2OC(CO)[C@@H](O)[C@H](O)[C@H]2O)OC=C2C(=O)OCCC21</smiles>

Sweroside (1)

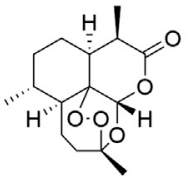

Artemisinin (2)

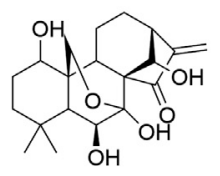

Oridonin (3)<smiles>C=C1CC[C@H]2[C@H](CC[C@@H](O)C2(C)CO)[C@H]1C/C=C1/C(=O)O[C@H](C)C[C@@H]1O</smiles>

Andrographolide (4) Ho

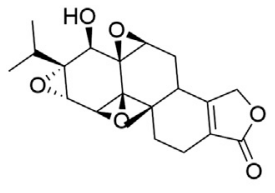

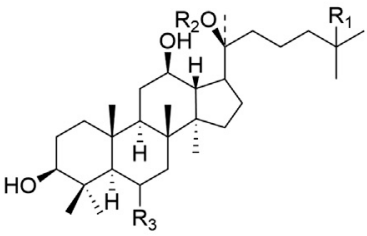

25-OCH3-PPD (6)

$\mathrm{R}_{1}=\mathrm{OCH}_{3} \quad \mathrm{R}_{2}=\mathrm{H} \mathrm{R}_{3}=\mathrm{H}$ OH

Triptolide (5) Compound $\mathrm{K}(7) \quad \mathrm{R}_{1}=\mathrm{H}_{2} \quad \mathrm{R}_{2}=$ glc $\mathrm{R}_{3}=\mathrm{H}$

Ginsenoside $\operatorname{Rgl}(8) \mathrm{R}_{1}=\mathrm{H}_{2}=$ glc $\mathrm{R}_{3}=\mathrm{glcO}$ -

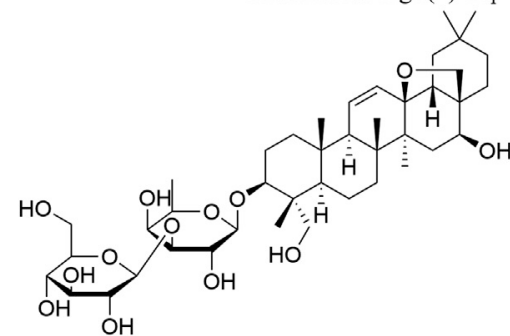<smiles>CC[C@H](CO)O[C@H]1OC[C@@H](O)[C@H](O)[C@H]1O[C@H]1O[C@H](C)[C@@H](O)[C@H](O)[C@H]1O</smiles>
ö̀<smiles>C1CCC2CCCCC2C1</smiles>

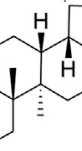<smiles>CCC(O)C1CC2OC(OCC3OC(OC(=O)C(C)(C)CC)C(O)C(O)C3O)C(O)C(C)C2O1</smiles>

FIGURE 2 | The chemical structures of terpenoids that inhibit NLRP3 inflammasome activation.

terpenoids (Figure 2), phenols, alkaloids, and other compounds (Figure 3), exhibit significant inhibitory activity against the NLRP3 inflammasome and can alleviate various fibrotic disorders, both in vitro and in vivo. Here, we describe all the phytochemicals that have been reported to ameliorate fibrosis via NLRP3 inflammasome inhibition in various cell and animal models, as well as the mechanisms underlying these inhibitory effects (Table 1).

\section{Terpenoids}

Terpenoids are a large group of widely distributed, bioactive natural products with diverse structures, and are composed of five-carbon (C5) units such as isopentenyl diphosphate or dimethylallyl diphosphate. Four main types of terpenoids have been reported to inhibit NLRP3 inflammasome activation: monoterpenoids (C10), sesquiterpenoids (C15), diterpenoids (C20), and triterpenoids (C30).

\section{Monoterpenoids}

Sweroside (1) is an iridoid monoterpene glucoside with obvious antifibrotic activity. It can effectively inhibit NLRP3 inflammasome activation in bone marrow-derived macrophages (BMDMs). Additionally, in a mouse model of nonalcoholic steatohepatitis (NASH) induced by a methionine-choline-deficient (MCD) diet, sweroside significantly attenuates NASH symptoms (including liver fibrosis) through the suppression of NLRP3 inflammasome activation in the liver, partially by inhibiting de novo synthesis of mitochondrial DNA (mtDNA), which can be oxidized to serve as the ultimate NLRP3 ligand, or at least a part of it, thus activating NLRP3 inflammasome complexes after exposure to LPS and NLRP3 activators (Zhong et al., 2018; Yang et al., 2020).

\section{Sesquiterpenoids}

Artemisinin (2) is a sesquiterpene endoperoxide known primarily as an effective medicine for the treatment of malaria. However, artemisinin and its derivatives have also shown potential antifibrotic value in recent studies (Wang et al., 2020c). Artemisinin can downregulate the NF- $\mathrm{B} / \mathrm{NLRP} 3$ pathway to attenuate tubulointerstitial fibrosis in subtotal nephrectomized $(\mathrm{SNx})$ rats, which is consistent with the results observed in Ang II-treated human kidney 2 (HK-2) cells (Wen et al., 2019).

\section{Diterpenoids}

Oridonin (3), andrographolide (4), and triptolide (5) are three diterpenoids exhibiting an antifibrotic effect via inhibiting NLRP3 inflammasome activation. In LPS-primed and nigericin-stimulated BMDMs, oridonin targets the NACHT domain of NLRP3 by binding to cysteine 279, thereby 
<smiles>[R]c1cc(O)cc(C(=O)c2cc(OC)c(OC)cc2O)c1-c1ccc(OC)c(-c2oc3cc(OC)c(OC)c(O)c3c(=O)c2OC)c1</smiles><smiles>[R7]Oc1cc(/C=C/c2ccc(O)cc2)cc(O[R2])c1</smiles>

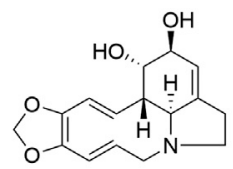

Lycorine (23)

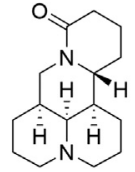

Matrine (24)<smiles>Cc1nc(C)c(C)nc1C</smiles>

Tetramethylpyrazine (25) Cinnamaldehyde (26)<smiles>CC1=C(/C=C/C(C)=C/C=C/C(C)=C/C=C/C=C(C)/C=C/C=C(C)/C=C/C2=C(C)C(=O)C(O)CC2(C)C)C(C)(C)CC(O)C1=O</smiles>

Astaxanthin (27)

FIGURE 3 | The chemical structures of phenols, alkaloids, and other compounds that inhibit NLRP3 inflammasome activation.

blocking NLRP3-NEK7 (a mitotic Ser/Thr kinase bridging adjacent NLRP3 subunits with bipartite interactions to activate the NLRP3 inflammasome) interaction and inhibiting NLRP3 inflammasome assembly and activation (He et al., 2018; Sharif et al., 2019). However, the specificity of oridonin for NLRP3 is dose-dependent, for it is insensitive to lipopolysaccharide (LPS)induced pro-IL-1 $\beta$, NLRP3 expression and TNF- $\alpha$ production but effective to inhibit the level of IL- $1 \beta$ at doses between 0.5 and $2.0 \mu \mathrm{M}$ (He et al., 2018). In addition, oridonin also inhibits TGF$\beta 1$ enhanced NLRP3 inflammasome activation in a human HSCs cell line, LX-2. Andrographolide can suppress LPS-induced IL-1 $\beta$ expression through NF-kB inhibition in fat-laden HepG2 cells and inflammasome disassembly (Cabrera et al., 2017). Triptolide exhibits significant efficacy in reducing the AngII-induced collagen production in cardiac fibroblasts by interrupting NLRP3/ASC interaction to inhibit NLRP3 inflammasome activation (Pan et al., 2019).

A series of research has also confirmed the efficacy of the diterpenoids above in vivo. In mice with $\mathrm{CCl}_{4}$-induced liver fibrosis, oridonin treatment significantly reduces the collagen deposition, HSCs (cells associated with hepatic fibrosis) activation, and recruitment of Kupffer cells, which may be related to its inhibitory effect on NLRP3 inflammasome activation in HSCs, highlighting the potential of the compound for use in the treatment of liver fibrosis. In mice with alcoholic steatohepatitis, andrographolide improves the liver fibrosis, decreases the hepatic fibrogenic proteins, such as connective tissue growth factor, collagen type I, alpha 1 chain and collagen IV, and suppresses the NLRP3 inflammasome activation (Cabrera et al., 2017). Recent studies have demonstrated the significant ability of triptolide to alleviate cardiac fibrosis through NLRP3 inflammasome inhibition. In chronic pressure-overloaded mice treated with triptolide, myocardial remodeling was attenuated through inhibition of the NLRP3 inflammasome and subsequently downstream inflammatory mediators such as IL- $1 \beta$ and IL-18, as well as inhibition of the TGF- $\beta 1$ pathway (Li et al., 2017b). Additionally, triptolide alleviates isoproterenol-induced cardiac fibrosis by inhibiting NLRP3/TGF $1 /$ Smad signaling in the mouse ventricle (Pan et al., 2019).

\section{Triterpenoids}

A myriad of triterpenoids and their derivatives, including the dammarane triterpenoids $25-\mathrm{OCH}_{3} \mathrm{PPD}(6)$, compound $\mathrm{K}(7)$, and ginsenoside $\operatorname{Rg} 1$ (8); the oleanane triterpenoid saponins glycyrrhizin (9) and saikosaponin A (10); and the lupane triterpenoid saponin anemoside B4 (11), exhibit markedly antifibrotic activity via inhibiting NLRP3 inflammasome activation.

In vitro study reveals that $25-\mathrm{OCH}_{3}-\mathrm{PPD}$ can decrease the expression of alpha smooth muscle actin ( $\alpha$-SMA) in TGF- $\beta$ stimulated HSC-T6 cell via inhibiting NLRP3 inflammasome 


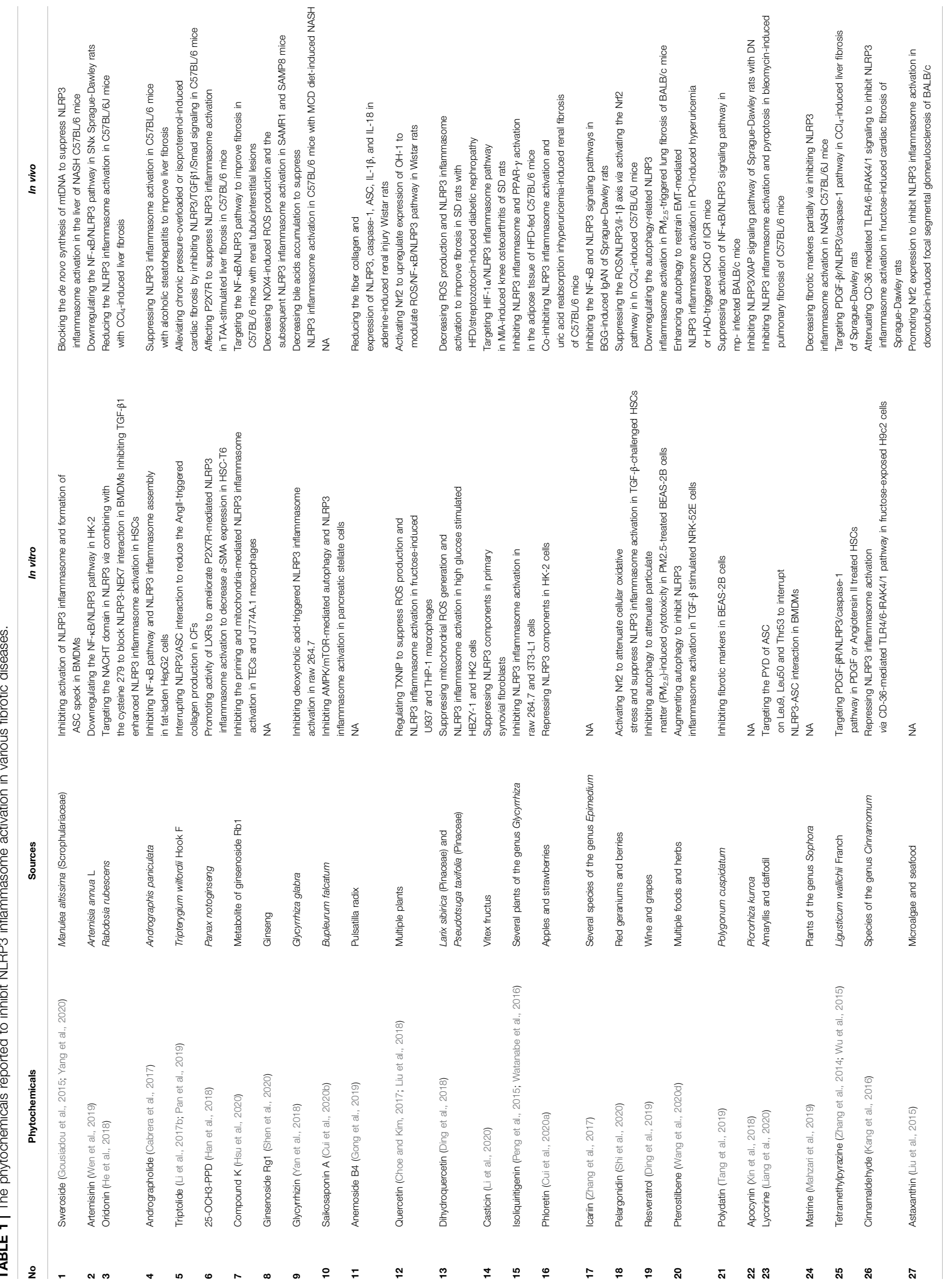


assembly, which is dependent on the inhibition of the purinergic $2 \mathrm{X} 7$ receptor $(\mathrm{P} 2 \mathrm{X} 7 \mathrm{R})$ induced by the promotion of liver $\mathrm{X}$ receptors (LXRs) (Han et al., 2018). Compound $\mathrm{K}$ possesses an inhibitory effect on the priming and mitochondria-mediated activation of the NLRP3 inflammasome as well as the STAT3 signaling pathway in renal tubular epithelial cells (TECs) under mechanically induced constant pressure (MICP) and murine J774A.1 macrophages. In Raw 264.7 macrophage cells, both glycyrrhizin and its metabolite glycyrrhetinic acid can significantly inhibit deoxycholic acid-induced NLRP3 inflammasome activation. Saikosaponin A exerts distinct inhibitory effects in suppressing the activation of pancreatic stellate cells, which are vital for the development of pancreatic fibrosis, by inhibiting autophagy and the NLRP3 inflammasome activation via the AMPK/mTOR pathway (Cui et al., 2020b).

Various studies have demomstrated the anti-fibrotic efficacy of triterpenoids in mice. Both $25-\mathrm{OCH}_{3}-\mathrm{PPD}$ and glycyrrhizin are effective in inhibiting hepatic fibrosis. In thioacetamide (TAA)-stimulated liver fibrosis of mice, 25- $\mathrm{OCH}_{3}-\mathrm{PPD}$ possesses the ability to reduce the expression levels of TIMP-1, MMP-13, and collagen I as well as the inhibition of ECM deposition, which is partially due to the regulation of NLRP3 inflammasome signaling pathway by affecting P2X7R activation (Han et al., 2018; Su et al., 2019). In mice with MCD diet-induced $\mathrm{NASH}$, glycyrrhizin and its metabolite glycyrrhetinic acid can significantly decrease bile acids accumulation, thus inhibiting the activation of NLRP3 inflammasome to attenuate liver fibrosis (Yan et al., 2018). Compound $\mathrm{K}$, ginsenoside Rg1, and anemoside $\mathrm{B} 4$ are reported to exhibit inhibitory effect on renal fibrosis. In mice with renal tubulointerstitial lesions, compound $\mathrm{K}$ reduces collagen III and total collagen content in the tubulointerstitial compartment, most likely by targeting the NF-кB/NLRP3 pathway (Hsu et al., 2020). Ginsenoside Rg1 can delay kidney aging and inhibit aging-related glomerular fibrosis by decreasing NOX4-induced ROS production and subsequently downregulating the expression of NLRP3 inflammasomeassociated proteins and production of the proinflammatory factor IL-1 $\beta$ (Shen et al., 2020). Anemoside B4 can reduce fiber collagen levels in the renal interstitium, lower the expression of NLRP3, caspase-1, ASC, IL-1 $\beta$, and IL-18, and enhance the expression of podocin and nephrin (two major podocyte proteins), suggesting that it exerts a protective effect on adenine-induced renal injury in rats (Gong et al., 2019).

\section{Phenols}

Phenolic compounds are secondary metabolites bearing one or more hydroxyl groups in the aromatic ring of their structures and are widely found in plants such as vegetables, fruits, and tea. Phenolic compounds are classified into multiple groups, including flavonoids, phenolic acids, and stilbenes, based on their structural characteristics (Lima et al., 2019).

\section{Flavonoids}

Flavonoids are naturally occurring and widely distributed plant secondary metabolites, and exhibit numerous biological functions, including antioxidative, antimicrobial, antiinflammatory, cardioprotective, and antifibrotic activities. The anti-inflammatory effect of flavonoids is mainly due to their ability to inhibit the production of proinflammatory mediators, such as TNF- $\alpha$, IL-1 $\beta$, IL-6, and IL-18, and regulate inflammationrelated signaling pathways, such as the TLR, NF- $\mathrm{B}, \mathrm{MAPK}$, and AP-1 pathways (Yi, 2018). An increasing number of flavonoids have been reported to exhibit anti-inflammatory and antifibrotic activities by inhibiting NLRP3 inflammasome activation, mainly including quercetin (12), dihydroquercetin (13), casticin (14), isoliquiritigenin (15), phloretin (16), icariin (17), and pelargonidin (18).

Pelargonidin, a type of anthocyanidin, can activate nuclear factor erythroid 2-like factor 2 (Nrf2) to attenuate cellular oxidative stress, thus suppressing the NLRP3 inflammasome activation and decreasing the COL1A1, TIMP1 production in TGF- $\beta$-challenged HSCs, suggesting that Nrf2-mediated ROS/ NLRP3/IL-1 $\beta$ signaling is involved in the anti-fibrotic effect of pelargonidin (Shi et al., 2020). Dihydroquercetin, a dihydroflavone compound, has been reported to attenuate the expression of renal fibrosis-associated proteins fibronectin and collagen IV in high glucose stimulated rat kidney mesangial cells (HBZY-1) and HK-2 cells, which may be associated with the suppression of mitochondrial ROS generation as well as NLRP3 inflammasome activation (Ding et al., 2018). Additionally, the naturally occurring dihydrogen chalcone flavonoid phloretin also inhibits the expression of NLRP3 as well as cleaved caspase-1, IL-1 $\beta$ and IL-18 protein in HK- 2 cells. Quercetin is a naturally occurring dietary flavonoid and acts as a supplement with excellent safety and bioavailability (Harwood et al., 2007). In vitro studies demonstrate that quercetin can suppress ROS production and subsequent NLRP3 inflammasome activation in fructose-induced U937 and THP-1 macrophages via the regulation of intracellular TXNIP, a protein that binds with NLRP3 to promote NLRP3 inflammasome activation (Choe and Kim, 2017). Casticin, also known as vitexicarpin, is effective at suppressing inflammatory factors (IL- $1 \beta$ and IL-18) and fibrogenic markers (TGF- $\beta$, COL1A1, and TIMP-1) via inhibiting NLRP3 components in primary synovial fibroblasts. Isoliquiritigenin, a flavonoid with a chalcone structure, inhibits TLR4-or Mincle-induced expression of fibrosis-associated genes in stromal vascular fraction from obese adipose tissue and macrophages in vitro via targeting the NLRP3 inflammasome.

The flavonoids also exhibit significantly anti-fibrotic effects in various disease models. Both quercetin and pelargonidin contribute to alleviating hepatic fibrosis by upregulating Nrf2 to inhibit NLRP3 inflammasome activation. In rats with acute alcoholic liver injury (AALI), quercetin activates Nrf2 to upregulate the expression of heme oxygenase-1 (HO-1), thus leading to a reduction in NLRP3 inflammasome activity and secretion of IL-1 $\beta$ and IL-18 (Liu et al., 2018). In $\mathrm{CCl}_{4}$-induced mice, pelargonidin suppress $a$-SMA and COL1A1 protein expression in liver via increasing Nrf2 levels and reducing oxidative stress, thereby inhibiting the NLRP3 inflammasome (Shi et al., 2020). Dihydroquercetin, phloretin, and icariin are all effective in ameliorating NLRP3 inflammasome-involved renal fibrosis. Dihydroquercetin treatment can decrease ROS production and NLRP3 inflammasome activation to improve 
fibrosis in rats with high fat diet (HFD)/streptozotocin-induced diabetic nephropathy (DN) (Ding et al., 2018). Phloretin can reduce hyperuricemia-induced renal inflammation, fibrosis, and mitochondrial stress in mice by co-inhibiting NLRP3 inflammasome activation and uric acid reabsorption (Cui et al., 2020a). In rats with bovine gamma globulin (BGG)induced $\operatorname{IgA}$ nephropathy (IgAN), icariin greatly decreases IgA deposition and glomerular fibrosis probably by inhibiting the NF$\kappa \mathrm{B}$ and NLRP3 signaling pathways (Zhang et al., 2017). In addition, Casticin exerts significant role in suppressing fibrogenic markers (TGF- $\beta$, COL1A1, and TIMP-1) and improving hypoxia in synovial tissue of rats with monoiodoacetic acid (MIA)-induced knee osteoarthritis (KOA) through the modulation of the hypoxia-inducible factor-1a (HIF1a), which is a transcriptional factor served to promote NLRP3 inflammasome activation ( $\mathrm{Li}$ et al., 2020). What is more, isoliquiritigenin can ameliorate inflammation and fibrosis in the adipose tissue of HFD-fed mice, and this effect is correlated with the inhibition of the NLRP3 inflammasome and PPAR $-\gamma$ activation (Watanabe et al., 2016).

\section{Stilbenes}

Stilbenes, comprising a subclass of polyphenolic compounds, possess many beneficial pharmacological properties, including neuroprotective, cardiovascular protective, antioxidative, and anti-inflammatory effects. Among the stilbenes, resveratrol (19), pterostilbene (20), and polydatin (21) have been reported to exert antifibrotic effects through the regulation of NLRP3 inflammasome activation.

Resveratrol, the most widely studied stilbene, possesses the ability to attenuate particulate matter $\left(\mathrm{PM}_{2.5}\right)$-induced cytotoxity in $\mathrm{PM}_{2.5}$-treated BEAS-2B cells via inhibiting autophagy, which is an evolutionarily conserved cellular process facilitating the clearance of NLRP3 activators such as $\beta$-amyloid and damaged mitochondria, thus suppressing the NLRP3 inflammasome activation (Nakahira et al., 2011; Cho et al., 2014). Pterostilbene, a dimethylated analog of resveratrol, can also markedly augment autophagy to inhibit NLRP3 inflammasome activation, thereby reducing the TGF- $\beta$ triggered expression of EMT markers including E-cadherin, fibronectin and $\alpha$-SMA in immortalized rat kidney proximal tubular epithelial cells (NRK-52E cells) (Wang et al., 2020d). Polydatin, a resveratrol glucoside, is able to decrease the expression of a-SMA, collagen I, and III in human lung epithelial BEAS-2B cells, however, the inhibitory effect of which can be reversed by overexpression of NLRP3.

The stilbenes also improve organ fibrosis in vivo. In models of potassium oxonate (PO)-induced hyperuricemia and high adenine diet (HAD)-induced chronic kidney disease (CKD), pterostilbene-triggered autophagy has an attenuating effect on the improvement of renal function and interstitial fibrosis via restraining EMT-mediated NLRP3 inflammasome activation (Wang et al., 2020d). Resveratrol is effective at alleviating lung inflammation and fibrosis caused by particulate matter $\left(\mathrm{PM}_{2.5}\right)$ via inhibiting autophagy-related NLRP3 inflammasome activation in BALB/c mice (Ding et al., 2019). In mice infected with Mycoplasma pneumomiae, polydatin treatment can suppress
NF-кB/NLRP3 signaling pathway activation, thereby attenuating lung inflammation and fibrosis.

\section{Other Phenolic Compounds}

Apocynin (22), a naturally occurring methoxy-substituted catechol has demonstrated an antifibrotic efficacy in major organs. Apocynin is widely used as an antioxidant via the inhibition of NOXs and the generation of superoxide anion, a ROS precursor (Touyz, 2008). Another study showed that apocynin hinders fibrosis in the renal cortex of rats with DN and decreases the expression of NLRP3 and X-linked inhibitor of apoptosis protein (XIAP) (Xin et al., 2018). XIAP functions as a caspase inhibitor, the expression of which is also decreased by the grape seeds with antioxidant activity, suggesting that XIAP may be involved in the NLRP3 inflammasome activation and renal fibrosis by regulating ROS (Guclu et al., 2015).

\section{Alkaloids}

Alkaloids are a group of nitrogen-containing organic secondary metabolites with diverse structures that are extensively distributed in plants, microbes, and even animals. Alkaloids have a wide variety of bioactive functions, including antitumor, antioxidant, anti-inflammatory, and immunoregulatory activities (Liu et al., 2019). Among the alkaloids, lycorine (23), matrine (24), and tetramethylpyrazine (25) have been shown to exert antifibrotic effects via inhibiting NLRP3 inflammasome activation.

Lycorine is an Amaryllidaceae alkaloid first identified as a potential candidate to treat cardiac fibrosis (Schimmel et al., 2020). It targets the pyrin domain (PYD) of ASC at Leu9, Leu50, and Thr53 to interrupt the interaction between NLRP3 and ASC, thus inhibiting NLRP3 inflammasome activation and pyroptosis in LPS-primed, nigericin-stimulated BMDMs (Liang et al., 2020). Tetramethylpyrazine, another important alkaloid to treat fibrosis, exerts the ability to suppress the enhanced expression of $a$-SMA and a1(I) procollagen in Angiotensin II-triggered HSCs, which is associated with the PDGF-mediated fibrotic pathways (Zhang et al., 2014). Additionally, tetramethylpyrazine exhibits inhibitory effect on the augmented expression of pro-IL-1 $\beta$, pro-IL-18, and cleaved IL- $1 \beta$ in platelet-derived growth factor (PDGF)-treated HSCs via regulating PDGF beta receptor (PDGF- $\beta$ R)/NLRP3/ caspase-1 pathway (Wu et al., 2015).

Various in vivo studies have demonstrated the role of alkaloids in treating fibrosis. In NASH mice fed an MCD diet, matrine decreases the expression of TGF- $\beta$, collagen I, and Smad3 by inhibiting the NLRP3 inflammasome, at least partially (Mahzari et al., 2019). In $\mathrm{CCl}_{4}$-induced liver injury in rats, tetramethylpyrazine possesses an antifibrotic role in alleviating liver injury and fibrogenesis through the involvement of PDGF$\beta R / N L R P 3$ signaling pathway. Lycorine is effective at decreasing the $a$-SMA, fibronectin and collagen content in bleomycininduced pulmonary fibrosis via inhibiting NLRP3 inflammasome activation and pyroptosis (Liang et al., 2020).

\section{Other Compounds}

Cinnamaldehyde (26) reduces the cellular TGF- $\beta, p-S m a d 2 / 3$ and Smad4 expression in fructose-exposed $\mathrm{H} 9 \mathrm{c} 2$ cells by repressing oxidative stress and ROS production, thereby inhibiting NLRP3 
TABLE 2 | Crude extracts from Traditional Chinese Herbs showed to modulate NLRP3 inflammasome activation in various fibrotic diseases.

\begin{tabular}{|c|c|c|}
\hline Crude extracts & Model System & Potential mechanism \\
\hline Salvianolates (Qiu et al., 2018) & Sprague-Dawley & Reducing the levels of IL-1 $\beta$ and IL-18 and escalating the expression of SIRT1 through TXNIP/NLRP3 pathway \\
\hline Danggui Buxue Tang (Wang et al., 2016) & Sprague-Dawley & Suppressing NLRP3 inflammasome activation \\
\hline Quamoclit angulata (Park et al., 2020) & C57BL/6 & Inhibiting NLRP3 inflammasome-dependent pathway \\
\hline Huangkui capsule (Han et al., 2019) & Sprague-Dawley & Depressing NLRP3 inflammasome and TLR4/NF-кB signaling activation \\
\hline
\end{tabular}

inflammasome activation via the CD-36-mediated TLR4/6-IRAK4/ 1 signaling pathway. Cinnamaldehyde protects human dental pulp cells against oxidative stress through the Nrf2/HO-1-dependent antioxidant response. Recent studies have demonstrated the pivotal role of $\mathrm{Nrf} 2$ in mediating the antioxidant effect of cinnamaldehyde, which significantly promotes Nrf2 activation, thereby inhibiting the TGF- $\beta 1$ and IL-13-dependent expression of periostin in fibroblasts (Mitamura et al., 2018; Wang et al., 2020b). Therefore, the cinnamaldehyde-induced activation of Nrf2 may also be responsible for the reduced ROS production and subsequent NLRP3 inflammasome inhibition.

Both cinnamaldehyde and astaxanthin (27) are effective at alleviating organ fibrosis by repressing oxidative stress. In rats fed with fructose, cinnamaldehyde represses cardiac fibrosis by supressing oxidative stress and ROS production, thereby inhibiting NLRP3 inflammasome activation via the CD-36mediated TLR4/6-IRAK4/1 signaling pathway (Kang et al., 2016). In a doxorubicin-induced mouse model of focal segmental glomerulosclerosis, astaxanthin elicits significant improvements in glomerular and interstitial fibrosis by promoting Nrf2 expression and inhibiting the NLRP3 inflammasome (Liu et al., 2015).

\section{CRUDE EXTRACTS THAT INHIBIT NLRP3 INFLAMMASOME ACTIVATION AND IMPROVE FIBROSIS}

Relatively few crude extracts obtained from natural plants have been reported to exhibit antifibrotic activity via NLRP3 inflammasome inhibition and mainly include salvianolate (Sal), Danggui Buxue Tang (DBT), Quamoclit angulata (QA), and Huangkui capsule (HKC) (Table 2).

Sal, the major water-soluble bioactive fraction of the Salvia miltiorrhiza, is composed of lithospermate B, rosmarinic acid, and lithospermic acid. In postmyocardial infarction (MI) model rats, Sal effectively reduces the left atrial levels of IL-1 $\beta$ and IL-18 and upregulates the expression of SIRT1, an enzyme that suppresses the TXNIP/NLRP3 inflammasome signaling pathway, thereby alleviating atrial interstitial fibrosis (Qiu et al., 2018).

DBT is a traditional Chinese medicine composed of two herbs, namely, Radix Astragali and Radix Angelicae Sinensis at a ratio of 5:1 (Gao et al., 2011). Studies have shown that total glucosides of DBT repress bleomycin-induced pulmonary fibrosis (Gao et al., 2011). In rats with unilateral ureteral obstruction (UUO), DBT treatment significantly reduces tubulointerstitial collagen deposition and tubulonterstitial fibrosis via suppressing NLRP3 inflammasome activation, thus protecting the rats with UUO (Wang et al., 2016).
QA is used in the treatment of diabetes and associated complications. In a mouse model of type 2 diabetes mellitus (T2DM), QA extract effectively attenuates renal fibrosis in a NLRP3 inflammasome-dependent manner (Park et al., 2020).

HKC, extracted from the Abelmoschus manihot flower, is a modern Chinese patent medicine composed mainly of flavonoids that is used for the treatment of chronic kidney diseases (Cai et al., 2017). In rat models of early DN, HKC significantly alleviates EMT effects by suppressing NLRP3 inflammasome and TLR4/ NF- $\mathrm{kB}$ signaling activation in the kidneys (Han et al., 2019).

\section{CONCLUSION AND FUTURE PERSPECTIVES}

NLRP3 is a major sensor of the innate immune system, the aberrant activation of which contributes to various fibrotic diseases, including $\mathrm{NASH}, \mathrm{CKD}, \mathrm{DN}$, and idiopathic pulmonary fibrosis (IPF). Identifying NLRP3 inhibitors in plant-derived natural products is of great importance for drug discovery as they are mostly safe, extensively distributed, and widely available. Among all the compounds, the diterpenoids may be the most promising candidates, for they possess combined inhibitory effect on NF- $\mathrm{kB}$ (the first signal of NLRP3 activation) pathway and NLRP3 inflammasome assembly (Hortelano et al., 2020). In this review, we have only summarized the natural products for which there is direct evidence that their antifibrotic effects are exerted via suppression of the NLRP3 inflammasome. Although other natural products, such as salidroside, berberine, and astragaloside IV, exhibit both NLRP3 inhibitory activity and antifibrotic activity (Dai et al., 2017; Dinesh and Rasool 2017; Guan et al., 2018; Qian et al., 2018; Li et al., 2019; Zhang et al., 2020), further investigation is needed to reveal whether the relationship is direct. In addition, the natural products currently reported to improve fibrogenesis through inhibiting NLRP3 inflammasome activation can only ameliorate fibrosis from early stages and cannot reverse existing fibrosis. Finally, in view of the NLRP3 inhibitors described above, further exploration is required for the following aspects. Firstly, although the binding sites for several natural products in NLRP3 inflammasome-related proteins have been identified, further studies are needed to reveal the mechanisms of action of potential NLRP3 inflammasome inhibitors and identify molecules that can selectively antagonize NLRP3 for the treatment of fibrotic diseases. Secondly, as a wide variation in the dose of compound used in different research may result in off-target effects and false-positive results, standardization of the dose for specific inhibition of NLRP3 will be of great significance for future studies. Thirdly, more efforts should be paid to the toxicity of the 
compounds in vivo, as unclear causes of side effects markedly restrict their application in clinic.

\section{AUTHOR CONTRIBUTIONS}

ND, BW, and XF prepared the draft. All the authors modified the draft.

\section{REFERENCES}

Artlett, C. M. (2012). The role of the NLRP3 inflammasome in fibrosis. Open Rheumatol. J. 6, 80-86. doi:10.2174/1874312901206010080

Baroja-Mazo, A., Martín-Sánchez, F., Gomez, A. I., Martínez, C. M., AmoresIniesta, J., Compan, V., et al. (2014). The NLRP3 inflammasome is released as a particulate danger signal that amplifies the inflammatory response. Nat. Immunol. 15, 738-748. doi:10.1038/ni.2919

Boucher, M. H., Cho, K., Kang, H. J., Jeon, E. Y., Kim, H. S., Kwon, H. J., et al. (2014). Autophagy in microglia degrades extracellular $\beta$-amyloid fibrils and regulates the NLRP3 inflammasome. Autophagy 10, 1761-1775. doi:10.4161/ auto. 29647

Bonnans, C., Chou, J., and Werb, Z. (2014). Remodelling the extracellular matrix in development and disease. Nat. Rev. Mol. Cell. Biol. 15, 786-801. doi:10.1038/ nrm3904

Cabrera, D., Wree, A., Povero, D., Solís, N., Hernandez, A., Pizarro, M., et al. (2017). Andrographolide ameliorates inflammation and fibrogenesis and attenuates inflammasome activation in experimental non-alcoholic steatohepatitis. Sci. Rep. 7, 3491. doi:10.1038/s41598-017-03675-z

Cai, H. D., Su, S. L., Qian, D. W., Guo, S., Tao, W. W., Cong, X. D., et al. (2017). Renal protective effect and action mechanism of Huangkui capsule and its main five flavonoids. J. Ethnopharmacol. 206, 152-159. doi:10.1016/j.jep.2017.02.046

Chen, G., Sun, L., Kato, T., Okuda, K., Martino, M. B., Abzhanova, A., et al. (2019). IL-1beta dominates the promucin secretory cytokine profile in cystic fibrosis. J. Clin. Invest. 129, 4433-4450. doi:10.1172/JCI125669

Choe, J. Y., and Kim, S. K. (2017). Quercetin and ascorbic acid suppress fructoseinduced NLRP 3 inflammasome activation by blocking intracellular shuttling of TXNIP in human macrophage cell lines. Inflammation 40, 980-994. doi:10. 1007/s10753-017-0542-4

Cui, L., Li, C., Zhuo, Y., Yang, L., Cui, N., Li, Y., et al. (2020). Saikosaponin A inhibits the activation of pancreatic stellate cells by suppressing autophagy and the NLRP3 inflammasome via the AMPK/mTOR pathway. Biomed. Pharmacother. 128, 110216. doi:10.1016/j.biopha.2020.110216

Cui, D., Liu, S., Tang, M., Lu, Y., Zhao, M., Mao, R., et al. (2020a). Phloretin ameliorates hyperuricemia-induced chronic renal dysfunction through inhibiting NLRP3 inflammasome and uric acid reabsorption. Phytomedicine 66, 153111. doi:10.1016/j.phymed.2019.153111

Dai, H., Jia, G., Lu, M., Liang, C., Wang, Y., and Wang, H. (2017). Astragaloside IV inhibits isoprenaline-induced cardiac fibrosis by targeting the reactive oxygen species/mitogen-activated protein kinase signaling axis. Mol. Med. Rep. 15, 1765-1770. doi: $10.3892 / \mathrm{mmr} .2017 .6220$

Dinesh, P., and Rasool, M. (2017). Berberine, an isoquinoline alkaloid suppresses TXNIP mediated NLRP3 inflammasome activation in MSU crystal stimulated RAW 264.7 macrophages through the upregulation of Nrf2 transcription factor and alleviates MSU crystal induced inflammation in rats. Int. Immunopharm. 44, 26-37. doi:10.1016/j. intimp.2016.12.031

Ding, S., Wang, H., Wang, M., Bai, L., Yu, P., and Wu, W. (2019). Resveratrol alleviates chronic "real-world" ambient particulate matter-induced lung inflammation and fibrosis by inhibiting NLRP3 inflammasome activation in mice. Ecotoxicol. Environ. Saf. 182, 109425. doi:10.1016/j.ecoenv.2019.109425

Ding, T., Wang, S., Zhang, X., Zai, W., Fan, J., Chen, W., et al. (2018). Kidney protection effects of dihydroquercetin on diabetic nephropathy through suppressing ROS and NLRP3 inflammasome. Phytomedicine 41, 45-53. doi:10.1016/j.phymed.2018.01.026

\section{FUNDING}

This work was supported by the National Natural Science Foundation of China under Grant Nos. 81671986 and 31872643, Hunan Provincial Key Laboratory for Special Pathogens Prevention and Control Foundation under Grant No. 2014-5, and the Hunan Province Cooperative Innovation Center for Molecular Target New Drug Study (2015-351).

Gao, J., Huang, Y., Li, P., Xu, D., Li, J., Liu, Y., et al. (2011). Antifibrosis effects of total glucosides of Danggui-Buxue-Tang in a rat model of bleomycin-induced pulmonary fibrosis. J. Ethnopharmacol. 136, 21-26. doi:10.1016/j.jep.2011.03. 013

Gong, Q., He, L. L., Wang, M. L., Ouyang, H., Gao, H. W., Feng, Y. L., et al. (2019). Anemoside B4 protects rat kidney from adenine-induced injury by attenuating inflammation and fibrosis and enhancing podocin and nephrin expression. Evid. Based. Complement. Alternat. Med. 2019, 8031039. doi:10.1155/2019/ 8031039

Gousiadou, C., Kokubun, T., Gotfredsen, C. H., and Jensen, S. R. (2015). Further iridoid glucosides in the genus Manulea (Scrophulariaceae). Phytochemistry 109, 43-48. doi:10.1016/j.phytochem.2014.10.004

Guan, C., Qiao, S., Lv, Q., Cao, N., Wang, K., Dai, Y., et al. (2018). Orally administered berberine ameliorates bleomycin-induced pulmonary fibrosis in mice through promoting activation of PPAR- $\gamma$ and subsequent expression of HGF in colons. Toxicol. Appl. Pharmacol. 343, 1-15. doi:10. 1016/j.taap.2018.02.001

Güçlü, A., Yonguç, N., Dodurga, Y., Gündoğdu, G., Güçlü, Z., Yonguç, T., et al. (2015). The effects of grape seed on apoptosis-related gene expression and oxidative stress in streptozotocin-induced diabetic rats. Ren. Fail. 37, 192-197. doi:10.3109/0886022X.2014.991996

Han, W., Ma, Q., Liu, Y., Wu, W., Tu, Y., Huang, L., et al. (2019). Huangkui capsule alleviates renal tubular epithelial-mesenchymal transition in diabetic nephropathy via inhibiting NLRP3 inflammasome activation and TLR4/NF-kappaB signaling. Phytomedicine 57, 203-214. doi:10.1016/j.phymed.2018.12.021

Han, X., Song, J., Lian, L. H., Yao, Y. L., Shao, D. Y., Fan, Y., et al. (2018). Ginsenoside 25-OCH3-PPD promotes activity of LXRs to ameliorate P2X7Rmediated NLRP3 inflammasome in the development of hepatic fibrosis. J. Agric. Food. Chem. 66, 7023-7035. doi:10.1021/acs.jafc.8b01982

Harwood, M., Danielewska-Nikiel, B., Borzelleca, J. F., Flamm, G. W., Williams, G. M., and Lines, T. C. (2007). A critical review of the data related to the safety of quercetin and lack of evidence of in vivo toxicity, including lack of genotoxic/ carcinogenic properties. Food. Chem. Toxicol. 45, 2179-205. doi:10.1016/j.fct. 2007.05.015

He, H., Jiang, H., Chen, Y., Ye, J., Wang, A., Wang, C., et al. (2018). Oridonin is a covalent NLRP3 inhibitor with strong anti-inflammasome activity. Nat. Commun. 9, 2550. doi:10.1038/s41467-018-04947-6

Hortelano, S., Gonzalez-Cofrade, L., Cuadrado, I., and de Las Heras, B. (2020). Current status of terpenoids as inflammasome inhibitors. Biochem. Pharmacol. 172, 113739. doi:10.1016/j.bcp.2019.113739

Hsu, W. H., Hua, K. F., Tuan, L. H., Tsai, Y. L., Chu, L. J., Lee, Y. C., et al. (2020). Compound $\mathrm{K}$ inhibits priming and mitochondria-associated activating signals of NLRP3 inflammasome in renal tubulointerstitial lesions. Nephrol. Dial. Transplant. 35, 74-85. doi:10.1093/ndt/gfz073

Huang, Q., Cai, W., Zhao, Y., Xu, H., Tang, H., Chen, D., et al. (2020). Lycorine ameliorates bleomycin-induced pulmonary fibrosis via inhibiting NLRP3 inflammasome activation and pyroptosis. Pharmacol. Res. 158, 104884. doi:10.1016/j.phrs.2020.104884

Kang, L. L., Zhang, D. M., Ma, C. H., Zhang, J. H., Jia, K. K., Liu, J. H., et al. (2016). Cinnamaldehyde and allopurinol reduce fructose-induced cardiac inflammation and fibrosis by attenuating CD36-mediated TLR4/6-IRAK4/1 signaling to suppress NLRP3 inflammasome activation. Sci. Rep. 6, 27460. doi:10.1038/srep27460

Kayagaki, N., Stowe, I. B., Lee, B. L., O'Rourke, K., Anderson, K., Warming, S., et al. (2015). Caspase-11 cleaves gasdermin D for non-canonical inflammasome signalling. Nature 526, 666-671. doi:10.1038/nature15541 
Ke, B., Shen, W., Fang, X., and Wu, Q. (2018). The NLPR3 inflammasome and obesityrelated kidney disease. J. Cell. Mol. Med. 22, 16-24. doi:10.1111/jcmm.13333

Kim, Y. G., Kim, S. M., Kim, K. P., Lee, S. H., and Moon, J. Y. (2019). The role of inflammasome-dependent and inflammasome-independent NLRP3 in the kidney. Cells 8, 1389. doi:10.3390/cells8111389

Li, R., Lu, K., Wang, Y., Chen, M., Zhang, F., Shen, H., et al. (2017). Triptolide attenuates pressure overload-induced myocardial remodeling in mice via the inhibition of NLRP3 inflammasome expression. Biochem. Biophys. Res. Commun. 485, 69-75. doi:10.1016/j.bbrc.2017.02.021

Li, R., Guo, Y., Zhang, Y., Zhang, X., Zhu, L., and Yan, T. (2019). Salidroside ameliorates renal interstitial fibrosis by inhibiting the TLR4/NF-kappaB and MAPK signaling pathways. Int. J. Mol. Sci. 20, 1103. doi:10.3390/ijms20051103

Li, X., Mei, W., Huang, Z., Zhang, L., Zhang, L., Xu, B., et al. (2020). Casticin suppresses monoiodoacetic acid-induced knee osteoarthritis through inhibiting HIF-1alpha/NLRP3 inflammasome signaling. Int. Immunopharmacol. 86, 106745. doi:10.1016/j.intimp.2020.106745

Liang, H., Xu, F., Zhang, T., Huang, J., Guan, Q., Wang, H., et al. (2018). Inhibition of IL-18 reduces renal fibrosis after ischemia-reperfusion. Biomed. Pharmacother. 106, 879-889. doi:10.1016/j.biopha.2018.07.031

Lima, M. C., Paiva de Sousa, C., Fernandez-Prada, C., Harel, J., Dubreuil, J. D., and de Souza, E. L. (2019). A review of the current evidence of fruit phenolic compounds as potential antimicrobials against pathogenic bacteria. Microb. Pathog. 130, 259-270. doi:10.1016/j.micpath.2019.03.025

Liu, C., Yang, S., Wang, K., Bao, X., Liu, Y., Zhou, S., et al. (2019). Alkaloids from traditional Chinese medicine against hepatocellular carcinoma. Biomed. Pharmacother. 120, 109543. doi:10.1016/j.biopha.2019.109543

Liu, G., Shi, Y., Peng, X., Liu, H., Peng, Y., and He, L. (2015). Astaxanthin attenuates adriamycin-induced focal segmental glomerulosclerosis. Pharmacology 95, 193-200. doi:10.1159/000381314

Liu, S., Tian, L., Chai, G., Wen, B., and Wang, B. (2018). Targeting heme oxygenase- 1 by quercetin ameliorates alcohol-induced acute liver injury via inhibiting NLRP3 inflammasome activation. Food. Funct. 9, 4184-4193. doi:10. 1039/c8fo00650d

Mahzari, A., Li, S., Zhou, X., Li, D., Fouda, S., Alhomrani, M., et al. (2019). Matrine protects against MCD-induced development of NASH via upregulating HSP72 and downregulating mTOR in a manner distinctive from metformin. Front. Pharmacol. 10, 405. doi:10.3389/fphar.2019.00405

Mangan, M. S. J., Olhava, E. J., Roush, W. R., Seidel, H. M., Glick, G. D., and Latz, E. (2018). Targeting the NLRP3 inflammasome in inflammatory diseases. Nat. Rev. Drug. Discov. 17, 588-606. doi:10.1038/nrd.2018.97

Mitamura, Y., Murai, M., Mitoma, C., and Furue, M. (2018). NRF2 activation inhibits both TGF-. Oxid. Med. Cell. Longev. 2018, 2475047. doi:10.1155/2018/ 2475047

Nakahira, K., Haspel, J. A., Rathinam, V. A., Lee, S. J., Dolinay, T., and Lam, H. C. (2011). Autophagy proteins regulate innate immune responses by inhibiting the release of mitochondrial DNA mediated by the NALP3 inflammasome. Nat. Immunol. 12, 222-230. doi:10.1038/ni.1980

Noronha, I. L., Fujihara, C. K., and Zatz, R. (2002). The inflammatory component in progressive renal disease-are interventions possible? Nephrol. Dial. Transplant. 17, 363-368. doi:10.1093/ndt/17.3.363

Otto, G. (2018). IL-1 $\beta$ switches on kidney fibrosis. Nat. Rev. Nephrol. 14, 475. doi:10.1038/s41581-018-0026-2

O’Brien, L. C., Mezzaroma, E., Van Tassell, B. W., Marchetti, C., Carbone, S., Abbate, A., et al. (2014). Interleukin-18 as a therapeutic target in acute myocardial infarction and heart failure. Mol. Med. 20, 221-229. doi:10.2119/ molmed.2014.00034

Pan, X. C., Liu, Y., Cen, Y. Y., Xiong, Y. L., Li, J. M., Ding, Y. Y., et al. (2019). Dual role of triptolide in interrupting the NLRP3 inflammasome pathway to attenuate cardiac fibrosis. Int. J. Mol. Sci. 20, 360. doi:10.3390/ijms20020360

Park, J. E., Lee, H., Rho, H., Hong, S. M., Kim, S. Y., and Lim, Y. (2020). Effect of Quamoclit angulata extract supplementation on oxidative stress and inflammation on hyperglycemia-induced renal damage in type 2 diabetic mice. Antioxidants 9, 459. doi:10.3390/antiox 9060459

Pelegrin, P., Alegre, F., and Feldstein, A. (2017). Inflammasomes in liver fibrosis. Semin. Liver Dis. 37, 119-127. doi:10.1055/s-0037-1601350

Peng, F., Du, Q., Peng, C., Wang, N., Tang, H., Xie, X., et al. (2015). A review: the Pharmacology of isoliquiritigenin. Phytother Res. 29, 969-977. doi:10.1002/ptr. 5348
Pinar, A. A., Scott, T. E., Huuskes, B. M., Tapia Cáceres, F. E., Kemp-Harper, B. K., and Samuel, C. S. (2020). Targeting the NLRP3 inflammasome to treat cardiovascular fibrosis. Pharmacol. Ther. 209, 107511. doi:10.1016/j. pharmthera.2020.107511

Qian, W., Cai, X., Qian, Q., Zhang, W., and Wang, D. (2018). Astragaloside IV modulates TGF-beta1-dependent epithelial-mesenchymal transition in bleomycin-induced pulmonary fibrosis. J. Cell Mol. Med. 22, 4354-4365. doi: $10.1111 /$ jcmm. 13725

Qiu, H., Liu, W., Lan, T., Pan, W., Chen, X., Wu, H., et al. (2018). Salvianolate reduces atrial fibrillation through suppressing atrial interstitial fibrosis by inhibiting TGF- $\beta 1 / \mathrm{Smad} 2 / 3$ and TXNIP/NLRP 3 inflammasome signaling pathways in post-MI rats. Phytomedicine 51, 255-265. doi:10.1016/j.phymed. 2018.09.238

Rockey, D. C., Bell, P. D., and Hill, J. A. (2015). Fibrosis--a common pathway to organ injury and failure. N. Engl. J. Med. 372, 1138-1149. doi:10.1056/ NEJMra1300575

Schimmel, K., Jung, M., Foinquinos, A., José, G. S., Beaumont, J., Bock, K., et al. (2020). Natural compound library screening identifies New molecules for the treatment of cardiac fibrosis and diastolic dysfunction. Circulation 141, 751-767. doi:10.1161/CIRCULATIONAHA.119.042559

Schroder, K., and Tschopp, J. (2010). The inflammasomes. Cell 140, 821-832. doi:10.1016/j.cell.2010.01.040

Shao, B. Z., Wang, S. L., Pan, P., Yao, J., Wu, K., Li, Z. S., et al. (2019). Targeting NLRP3 inflammasome in inflammatory bowel disease: putting out the fire of inflammation. Inflammation 42, 1147-1159. doi:10.1007/s10753-019-01008-y

Shao, Q., He, L. L., Wang, M. L., Ouyang, H., Gao, H. W., Feng, Y. L., et al. (2019). Anemoside B4 protects rat kidney from adenine-induced injury by attenuating inflammation and fibrosis and enhancing podocin and nephrin expression. Evid. Based. Complement. Alternat. Med. 2019, 8031039. doi:10.1155/2019/ 8031039

Sharif, H., Wang, L., Wang, W. L., Magupalli, V. G., Andreeva, L., Qiao, Q., et al. (2019). Structural mechanism for NEK7-licensed activation of NLRP3 inflammasome. Nature 570, 338-343. doi:10.1038/s41586-019-1295-Z

Shen, X., Dong, X., Han, Y., Li, Y., Ding, S., Zhang, H., et al. (2020). Ginsenoside Rg1 ameliorates glomerular fibrosis during kidney aging by inhibiting NOX4 and NLRP3 inflammasome activation in SAMP8 mice. Int. Immunopharm. 82, 106339. doi:10.1016/j.intimp.2020.106339

Shi, Y. S., Li, X. X., Li, H. T., and Zhang, Y. (2020). Pelargonidin ameliorates $\mathrm{CCl}_{4-}$ induced liver fibrosis by suppressing the ROS-NLRP3-IL-1 $\beta$ axis via activating the Nrf2 pathway. Food. Funct. 11, 5156-5165. doi:10.1039/d0fo00660b

Strutz, F. (1995). Novel aspects of renal fibrogenesis. Nephrol. Dial. Transplant. 10, $1526-1532$.

Su, G. Y., Li, Z. Y., Wang, R., Lu, Y. Z., Nan, J. X., Wu, Y. L., et al. (2019). Signaling pathways involved in p38-ERK and inflammatory factors mediated the antifibrosis effect of AD-2 on thioacetamide-induced liver injury in mice. Food. Funct. 10, 3992-4000. doi:10.1039/c8fo02405g

Swanson, K. V., Deng, M., and Ting, J. P. (2019). The NLRP3 inflammasome: molecular activation and regulation to therapeutics. Nat. Rev. Immunol. 19, 477-489. doi:10.1038/s41577-019-0165-0

Tang, J., Li, Y., Wang, J., Wu, Q., and Yan, H. (2019). Polydatin suppresses the development of lung inflammation and fibrosis by inhibiting activation of the NACHT domain-, leucine-rich repeat-, and pyd-containing protein 3 inflammasome and the nuclear factor-kappaB pathway after Mycoplasma pneumoniae infection. J. Cell. Biochem. 120, 10137-10144. doi:10.1002/jcb. 28297

Touyz, R. M. (2008). Apocynin, NADPH oxidase, and vascular cells: a complex matter. Hypertension 51, 172-174. doi:10.1161/HYPERTENSIONAHA.107. 103200

Wang, D., Duncan, B., Li, X., and Shi, J. (2020a). The role of NLRP3 inflammasome in infection-related, immune-mediated and autoimmune skin diseases. J. Dermatol. Sci. 98, 146-151. doi:10.1016/j.jdermsci.2020.03.001

Wang, L., Ma, J., Guo, C., Chen, C., Yin, Z., Zhang, X., et al. (2016). Danggui Buxue Tang attenuates tubulointerstitial fibrosis via suppressing NLRP3 inflammasome in a rat model of unilateral ureteral obstruction. BioMed Res. Int. 2016, 9368483. doi:10.1155/2016/9368483

Wang, P., Yang, Y., Wang, D., Yang, Q., Wan, J., Liu, S., et al. (2020b). Cinnamaldehyde ameliorates vascular dysfunction in diabetic mice by activating Nrf2. Am. J. Hypertens. 33, 610-619. doi:10.1093/ajh/hpaa024 
Wang, W., Wang, X., Chun, J., Vilaysane, A., Clark, S., French, G., et al. (2013). Inflammasome-independent NLRP3 augments TGF-beta signaling in kidney epithelium. J. Immunol. 190, 1239-1249. doi:10.4049/jimmunol.1201959

Wang, Y. J., Chen, Y. Y., Hsiao, C. M., Pan, M. H., Wang, B. J., Chen, Y. C., et al. (2020d). Induction of autophagy by pterostilbene contributes to the prevention of renal fibrosis via attenuating NLRP3 inflammasome activation and epithelial-mesenchymal transition. Front. Cell. Dev. Biol. 8, 436. doi:10.3389/ fcell.2020.00436

Wang, Y., Wang, Y., You, F., and Xue, J. (2020c). Novel use for old drugs: the emerging role of artemisinin and its derivatives in fibrosis. Pharmacol. Res. 157, 104829. doi:10.1016/j.phrs.2020.104829

Watanabe, Y., Nagai, Y., Honda, H., Okamoto, N., Yamamoto, S., Hamashima, T., et al. (2016). Isoliquiritigenin attenuates adipose tissue inflammation in vitro and adipose tissue fibrosis through inhibition of innate immune responses in mice. Sci. Rep. 6, 23097. doi:10.1038/srep23097

Wen, Y., Pan, M. M., Lv, L. L., Tang, T. T., Zhou, L. T., Wang, B., et al. (2019). Artemisinin attenuates tubulointerstitial inflammation and fibrosis via the NF$\mathrm{kB} / \mathrm{NLRP} 3$ pathway in rats with 5/6 subtotal nephrectomy. J. Cell. Biochem. 120, 4291-4300. doi:10.1002/jcb.27714

Wree, A., McGeough, M. D., Pena, C. A., Schlattjan, M., Li, H., Inzaugarat, M. E., et al. (2014). NLRP3 inflammasome activation is required for fibrosis development in NAFLD. J. Mol. Med. 92, 1069-1082. doi:10.1007/s00109-014-1170-1

Wu, X., Zhang, F., Xiong, X., Lu, C., Lian, N., Lu, Y., et al. (2015). Tetramethylpyrazine reduces inflammation in liver fibrosis and inhibits inflammatory cytokine expression in hepatic stellate cells by modulating NLRP3 inflammasome pathway. IUBMB Life 67, 312-321. doi:10.1002/iub.1348

Wynn, T. A., and Ramalingam, T. R. (2012). Mechanisms of fibrosis: therapeutic translation for fibrotic disease. Nat. Med. 18, 1028-1040. doi:10.1038/nm.2807

Xiao, H., Li, H., Wang, J. J., Zhang, J. S., Shen, J., An, X. B., et al. (2018). IL-18 cleavage triggers cardiac inflammation and fibrosis upon beta-adrenergic insult. Eur. Heart J. 39, 60-69. doi:10.1093/eurheartj/ehx261

Xin, R., Sun, X., Wang, Z., Yuan, W., Jiang, W., Wang, L., et al. (2018). Apocynin inhibited NLRP3/XIAP signalling to alleviate renal fibrotic injury in rat diabetic nephropathy. Biomed. Pharmacother. 106, 1325-1331. doi:10.1016/j.biopha. 2018.07.036

Yan, T., Wang, H., Cao, L., Wang, Q., Takahashi, S., Yagai, T., et al. (2018). Glycyrrhizin alleviates nonalcoholic steatohepatitis via modulating bile acids and meta-inflammation. Drug Metab. Dispos. 46, 1310-1319. doi:10.1124/dmd. 118.082008

Yang, G., Jang, J. H., Kim, S. W., Han, S. H., Ma, K. H., Jang, J. K., et al. (2020). Sweroside prevents non-alcoholic steatohepatitis by suppressing activation of the NLRP3 inflammasome. Int. J. Mol. Sci. 21. doi:10.3390/ijms21082790

Yi, Y. S. (2018). Regulatory roles of flavonoids on inflammasome activation during inflammatory responses. Mol. Nutr. Food Res. 62, e1800147. doi:10.1002/mnfr. 201800147

Zhang, L., Wang, X. Z., Li, Y. S., Zhang, L., and Hao, L. R. (2017). Icariin ameliorates IgA nephropathy by inhibition of nuclear factor kappa b/Nlrp3 pathway. FEBS. Open. Bio. 7, 54-63. doi:10.1002/2211-5463.12161

Zhang, X., Mei, W., Huang, Z., Zhang, L., Zhang, L., Xu, B., et al. (2020). Casticin suppresses monoiodoacetic acid-induced knee osteoarthritis through inhibiting HIF-1a/NLRP3 inflammasome signaling. Int. Immunopharm. 86, 106745. doi:10.1016/j.intimp.2020.106745

Zhang, X., Kuang, G., Wan, J., Jiang, R., Ma, L., Gong, X., et al. (2020). Salidroside protects mice against $\mathrm{CCl}_{4}$-induced acute liver injury via down-regulating CYP2E1 expression and inhibiting NLRP3 inflammasome activation. Int. Immunopharm. 85, 106662. doi:10.1016/j.intimp.2020.106662

Zhang, X., Zhang, F., Kong, D., Wu, X., Lian, N., Chen, L., et al. (2014). Tetramethylpyrazine inhibits angiotensin II-induced activation of hepatic stellate cells associated with interference of platelet-derived growth factor beta receptor pathways. FEBS J. 281, 2754-2768. doi:10.1111/febs.12818

Zhong, Z., Liang, S., Sanchez-Lopez, E., He, F., Shalapour, S., Lin, X. J., et al. (2018). New mitochondrial DNA synthesis enables NLRP3 inflammasome activation. Nature 560, 198-203. doi:10.1038/s41586-018-0372-Z

Conflict of Interest: The authors declare that the research was conducted in the absence of any commercial or financial relationships that could be construed as a potential conflict of interest.

Copyright (c) 2020 Ding, Wei, Fu, Wang and Wu. This is an open-access article distributed under the terms of the Creative Commons Attribution License (CC BY). The use, distribution or reproduction in other forums is permitted, provided the original author(s) and the copyright owner(s) are credited and that the original publication in this journal is cited, in accordance with accepted academic practice. No use, distribution or reproduction is permitted which does not comply with these terms. 